\title{
HOMOGENEOUS ALMOST COMPLEX SPACES OF POSITIVE CHARACTERISTIC
}

\section{SAMUEL PASIENCIER}

In this paper we give a necessary and sufficient condition for a homogeneous space of positive Euler characteristic to admit an invariant almost complex structure (i.a.c.s.), Theorem 4.1. A classification of these spaces has already been accomplished by R. Hermann [3], but no characterization of them has been given, to date. This work is a portion of the author's dissertation directed by H. C. Wang.

1. Let $G$ be a compact, connected Lie group, and $L$ a closed subgroup of $G, \mathcal{S}$ and $\&$ their Lie algebras. Then as is well known, $L$ is reductive in $G$ and we may write $\mathcal{G}=\mathfrak{L}+\mathfrak{T}$, where Ad $L: \mathfrak{T} \rightarrow \mathfrak{M}$. Moreover by homogeneity, a necessary and sufficient condition that there exist an almost complex structure on $G / L$, is that there exist a linear transformation $J: \mathfrak{M} \rightarrow \mathfrak{M}$, such that $J^{2}=-I$ ( $I$, the identity), and such that $\operatorname{Ad} l J=J$ Ad $l$ for all $l \in L$. See for example, A. Frölicher [2].

2. We give first a sufficient condition in the general case.

THEOREM 2.1. Let $Z$ be a closed, abelian subgroup of a compact Lie group $G$, and $L$ the identity component of the centralizer of $Z$ in $G$. If each 1-dimensional subspace of $\mathcal{G}$ invariant by $\operatorname{Ad} Z$ is pointwise fixed, then $G / L$ has an i.a.c.s.

Proof. $Z$ being compact and abelian, Ad $Z$ is completely reducible; with notation as in 1 , there cannot exist any 1-dimensional subspaces of $\mathfrak{T}$ reducing $\operatorname{Ad} Z$, for if $X \neq 0$ belonged to such a subspace, then by hypothesis we would have $\operatorname{Ad} z(X)=X$ for all $z \in Z$, and thus $X \in \mathcal{L}$, which is impossible.

If $C \mathscr{T}$ denotes the complexification of $\Re$, and $\operatorname{Ad} Z$ and $\operatorname{Ad} L$ are extended by linearity to $C \mathscr{T}$, then we know by Schur's Lemma that Ad $Z$ acting on $C \mathscr{T}$ has a weight vector $v_{i}$. That is, $\operatorname{Ad} z\left(v_{i}\right)=\psi_{i}(z) v_{i}$ for all $z \in Z, \psi_{i}$ being a character on $Z$. Moreover, since $\operatorname{Ad} Z$ is real, Ad $z\left(\bar{v}_{i}\right)=\bar{\psi}_{i}(z) \bar{v}_{i}$, thus the weight vectors and roots appear in conjugate pairs.

Let $v_{1}, \cdots, v_{n}, \bar{v}_{1}, \cdots, \bar{v}_{n}$ be the weight vectors with characters $\psi_{1}, \cdots, \psi_{n}, \bar{\psi}_{1}, \cdots, \bar{\psi}_{n}$, where some of the $\psi_{i}$ 's may be identical. If

Received by the editors September 4, 1962 and, in revised forms, December 3, 1962 and April 9, 1963. 
we let $\vartheta$ be the subspace of $C \mathscr{T}$ spanned by the $v_{i}$ 's, then each $x \in \mathfrak{N}$ has a unique expression of the form $x=v+\bar{v}$, where $v \in \mathcal{V}$ and $\bar{v} \in \bar{v}$. For putting $x_{j}=v_{j}+\bar{v}_{j}$, and $x_{j}^{\prime}=i v_{j}-i \bar{v}_{j}$ the $x_{j}^{\prime}$ 's and $x_{j}^{\prime}$ 's span the irreducible subspaces of $\mathfrak{T}$. And thus any $x \in \mathscr{T}$ has a unique expression

$$
x=\sum a_{j} x_{j}+\sum b_{j} x_{j}^{\prime}
$$

so

$$
x=\sum \lambda_{j} v_{j}+\sum \bar{\lambda}_{j} \bar{v}_{j}
$$

Furthermore, $v$ is invariant by $\operatorname{Ad} L$, for if $z \in Z, l \in L$, then Ad $z \operatorname{Ad} l\left(v_{i}\right)=\operatorname{Ad} l$ Ad $z\left(v_{i}\right)=\psi_{i}(z)$ Ad $l\left(v_{i}\right)$, that is, Ad $l\left(v_{i}\right)$ belongs to the character $\psi_{i}$, and by hypothesis there can be no real characters, thus $\psi_{i} \neq \bar{\psi}_{j}$. Now defining $J: \mathfrak{T} \rightarrow \mathfrak{M}$ by $J(x)=J(v+\bar{v})=i v-i \bar{v}$, defines an i.a.c.s. on $G / L$.

CoROllaRy 2.1. If the number of connected components in $\operatorname{Ad} Z$ is odd, then $G / L$ has an i.a.c.s., $G, L, Z$ as above.

Proof. Suppose there exists a 1-dimensional subspace of $\mathfrak{T}$, with basis $X$, reducing $\operatorname{Ad} Z$, then for each $z \in Z, \operatorname{Ad} z(X)=\psi(z) X$, where $\psi(z)$ is a homomorphism of $Z \rightarrow Z_{2}=\{+1,-1\}$. By continuty $\psi$ is constant on components of $\mathrm{Ad} z$, and takes the value +1 on the identity component $(\operatorname{Ad} z)^{0}$. It thus induces a homomorphism $\psi: \operatorname{Ad} z /(\operatorname{Ad~} z)^{0} \rightarrow Z_{2}$, but $\operatorname{Ad} z /(\operatorname{Ad} z)^{0}$ is a finite group of odd order, therefore its image is identically 1 , so that $\psi \equiv 1$, and thus $\operatorname{Ad} z$ is pointwise fixed on each 1-dimensional subspace reducing it.

3. Next we consider the case that $L$ is semi-simple.

Lemмa 3.1. Let $L, H$ be two closed subgroups of $G, L \subset H \subset G$. And suppose that $G / L$ has an i.a.c.s. defined by $J: \mathfrak{T} \rightarrow \mathfrak{T}$, where $\mathcal{G}=\mathfrak{L}+\mathfrak{T}$. If further $\mathfrak{H}=\mathscr{L}+\mathfrak{M}_{1}, \mathfrak{M}_{1} \subset \mathfrak{M}$, and $J\left(\mathfrak{M}_{1}\right)=\mathfrak{M}_{1}$, then $H / L$ has an i.a.c.s.

Proof. Since $H$ is a subgroup of $G, \operatorname{Ad} L: \Re_{1} \rightarrow \Re_{1}$. Thus applying 1, we see that $H / L$ has an i.a.c.s.

Lemma 3.2. Let $L$ be a closed semi-simple subgroup of $G$, of maximal rank, such that $G / L$ has an i.a.c.s.; then $L$ is not a symmetric subgroup.

Proof. Let $\mathcal{G}=\mathfrak{L}+\mathfrak{N}$, and $J: \mathfrak{N} \rightarrow \mathfrak{M}$ define the i.a.c.s. as in $\$ 1$. Since the torsion tensor of $J$ is given by

$$
\mathcal{F}(X, Y)=[J X, J Y]-J[J X, Y]-J[X, J Y]+[X, Y] \bmod \mathfrak{L}
$$

for $X, Y \in \mathfrak{N}[2]$, if we assume $G / L$ is symmetric $\mathcal{F}(X, Y) \equiv 0$, for 
symmetric implies $[\mathfrak{T}, \mathfrak{T}] \subset L$. Thus $J$ defines a complex structure on $G_{0} / L_{0}$ (where subscripts denote identity components), but then a classical result of $\mathrm{E}$. Cartan says that $L_{0}$ (and thus $L$ ) cannot be semisimple.

LEMMA 3.3. If $G / L$ has an i.a.c.s. and $L$ is connected semi-simple of maximal rank, $Z$ the center of $L$; then $\operatorname{Ad}(Z)$ acting on $\mathfrak{T C}$ has no 1dimensional invariant subspaces.

Proof. Suppose by contradiction that $R \subset \mathfrak{T}$ is 1 -dimensional and reduces $\operatorname{Ad}(Z)$. Let $x \in R, x \neq 0$, then

$\operatorname{Ad} z(x)=\psi(z) x, z \in Z$, and $\psi$ is a real character on $Z$.

Since $Z$ is compact, either $\psi(Z) \equiv 1$, or $\psi(Z)=\{+1,-1\}$. From BoreldeSiebenthal [1], we know that $L$ is the connected component of the centralizer of its own center $Z$, and thus $\mathscr{L}=\{u: u \in \mathcal{G}$, Ad $Z u=u\}$. If $\psi(Z) \equiv 1$, then $R \subset \mathcal{L}$, which is impossible. Thus $\psi(Z)=\{1,-1\}$.

Let $Q=\{y: y \in \Re$, Ad $z(y)=\psi(z) y, z \in Z\}$ be the weight space corresponding to the character $\psi$. Then $J(\mathcal{Q})=\mathcal{Q}$, for if $y \in \mathcal{Q}$,

$$
\operatorname{Ad} z J(y)=J \cdot \operatorname{Ad} z(y)=J \psi(z) y=\psi(z) J y .
$$

Furthermore, $\operatorname{Ad} L(Q)=Q$, for if $y \in Q, l \in L$, then

$$
\operatorname{Ad} z \operatorname{Ad} l y=(\operatorname{Ad} l) \psi(z) y=\psi(z) \operatorname{Ad} l y .
$$

But then

$$
[\mathfrak{L}, \Theta] \subset \mathcal{Q} \text {. }
$$

Finally if $y, y^{\prime} \in Q, \operatorname{Ad} z\left[y, y^{\prime}\right]=\psi(z)^{2}\left[y, y^{\prime}\right]=\left[y, y^{\prime}\right]$, that is, $\left[y, y^{\prime}\right]$ is fixed under all $\mathrm{Ad} z$, so that

$$
\left[y, y^{\prime}\right] \in \mathcal{L} \text {, that is }[\mathcal{Q}, \Theta] \subset \mathcal{L} .
$$

Therefore, $\mathcal{H}=\mathscr{L}+\mathcal{Q}$ is a subalgebra and $H / L$ is a symmetric space, $H=\exp \mathcal{H}$. Since $H$ is of maximal rank, $H$ is closed by the theorem in $\$ 8$ of [1]. By Lemma $3.1, H / L$ has an invariant almost complex structure, and $H / L$ is nontrivial since $Q \neq 0$, for $R \subset Q$. This contradicts Lemma 3.2, thus Lemma 3.3 is proven.

LEMmA 3.4. Under the hypothesis of Lemma 3.3, $\operatorname{Ad}(Z)$ cannot be a group of order $2^{k}, k>0$.

Proof. (a) If $k=1, G / L$ is symmetric and this is impossible by Lemma 3.2.

(b) Suppose the lemma is true for all $k \leqq n$, and consider the case that $\operatorname{Ad} Z$ is of order $2^{n+1}$. Since $\operatorname{Ad} Z$ contains a subgroup of order 2, 
there exists an element $z_{0} \in Z$ such that $\operatorname{Ad} z_{0} \neq 1$ and $\left(\operatorname{Ad} z_{0}\right)^{2}=1$. Let $G^{*}$ be the 1 -component of the centralizer of $z_{0}$ in $G$. Then $L \subset G^{*}$, and we have

$$
\mathcal{S}=\mathfrak{L}+\mathfrak{M}, \quad \mathfrak{M}=Q_{1}+Q_{2}, \quad \mathcal{S}^{*}=\mathfrak{L}+Q_{1},
$$

where $\left.\operatorname{Ad} z_{0}\right|_{Q_{1}}=1$ and $\left.\operatorname{Ad} z_{0}\right|_{Q_{2}}=-1, G^{*}$ being the Lie algebra of $G^{*}$. Moreover $J\left(Q_{1}\right)=Q_{1}$ for if $m \in Q_{1},(\operatorname{Ad} z) J(m)=J(\operatorname{Ad} z) m=J(m)$. Similarly we show that $\operatorname{Ad} L\left(Q_{1}\right)=Q_{1}$.

$G^{*} / L$ has i.a.c.s. by Lemma 3.1. But since $L \subset G^{*}, Z$ is the center of $L$ in $G^{*}$. Since $\operatorname{Ad} z_{0}\left|g^{*}=1, \operatorname{Ad} Z\right|_{g^{*}}$ is of order $2^{m}, m \leqq n$. Thus by the inductive hypothesis $m=0$ and $G^{*}=L$. But then $G / L$ is a symmetric space, contradicting Lemma 3.2. Thus $G / L$ cannot have invariant almost complex structure, and the lemma is proved.

TheOREM 3.5. Let $G$ be a compact connected Lie group and $L$ a connected semi-simple subgroup of $G$ of maximal rank; then $G / L$ has invariant almost complex structure if and only if $L$ is the identity component of the centralizer in $G$ of a finite subgroup $F$, of its center, such that $\operatorname{Ad}(F)$ is of odd order.

Proof. The sufficiency of this condition was proved in Theorem 2.1. To prove the necessity, let $Z$ be the center of $L$; then by the fundamental theorem of Abelian groups we may write $\operatorname{Ad} Z=\operatorname{Ad} F_{1}$ $\times \operatorname{Ad} F_{2}$, where $\operatorname{Ad} F_{1}$ is of odd order and $\operatorname{Ad} F_{2}$ is of order $2^{k}$.

Let $G_{1}$ be the identity component of the centralizer of $F_{1}$ in $G$. We shall show that $G_{1}=L$. Suppose on the contrary that $L_{\neq}^{\complement} G_{1}$. Then $G_{1} / L$ has i.a.c.s. by Lemma 3.1. If $\operatorname{Ad}\left(F_{2}\right): \mathcal{G}_{1} \rightarrow \mathcal{G}_{1}$ is identically 1 , then we are done because $\mathcal{G}_{1}$ centralizes both $F_{1}$ and $F_{2}$ and by the result of Borel and de Siebenthal [1], $G_{1}=L$. But $\operatorname{Ad}\left(F_{1}\right)$ is identically 1 on $G_{1}$, and $\operatorname{Ad}\left(F_{2}\right)$, if nontrivial on $G_{1}$, must be of order $2^{n}$, for some $n>1$. But then Ad $Z$ acting on $\mathcal{G}_{1}$ would be of order $2^{n}$, which is impossible. Therefore $\operatorname{Ad}\left(F_{2}\right) \equiv 1$ on $\mathcal{G}_{1}$.

4. Theorem 4.1. Let $G$ be a compact connected Lie group, $L$ a connected subgroup of maximal rank; then $G / L$ possesses an invariant almost complex structure if and only if $L$ is the connected component of the centralizer in $G$ of a finite subgroup $F$, of its center, such that $\operatorname{Ad}(F)$ is a finite group of odd order.

The sufficiency of this condition follows from Corollary 2.1. In this general case when $L$ is not necessarily semi-simple, we proceed by proving the following two lemmas.

Lemma 4.2. Let $G$ be compact, connected, $L$ a connected subgroup of maximal rank such that $G / L$ has an i.a.c.s.; if $L=T^{a} \times L_{1}$, local direct 
product, where $T^{a}$ is toral and $L_{1}$ is connected semi-simple, then $L$ is the centralizer in $G$ of $T_{a} \cup F_{1}$, where $F_{1}$ is a finite subgroup of $L$, such that $\operatorname{Ad}\left(F_{1}\right)$ is of odd order.

Proof. $L=T^{a} \times L_{1}, \mathfrak{L}=R^{a} \oplus \mathfrak{L}_{1}$, let $G_{2}=$ centralizer of $T^{a}$ in $G$, then $G_{2}$ is a connected subgroup of maximal rank in $G$, and

$$
G_{2}=T^{a} \times G_{3}, \quad g_{2}=R^{a} \oplus \mathcal{G}_{3}, \quad \text { where } G_{3} \text { is closed in } G_{2} .
$$

By hypothesis there exists $J$, commuting with $\operatorname{Ad}(L)$ such that

$$
J: \mathcal{G}_{2} / \mathfrak{L} \rightarrow \mathcal{G}_{2} / \mathfrak{L}
$$

but $\mathfrak{L}_{1} \subset \mathfrak{L}$, and there exists an isomorphism

$$
\phi: \mathcal{G}_{2} / \mathfrak{L} \rightarrow \mathcal{G}_{3} / \mathfrak{L}_{1} .
$$

Define $J^{\prime}$ on $\mathcal{G}_{3} / \mathscr{L}_{1}$ by $J^{\prime}=\phi J \phi^{-1}$, then $J^{\prime}$ defines an almost complex structure on $G_{3} / L_{1}$. Then, by $3.5, L_{1}$ is the connected component of the centralizer in $G_{3}$ of a finite subgroup $F_{1}$ of its center, such that $\operatorname{Ad}\left(F_{1}\right)$ is of odd order.

Now, if $x \in L$, then $x=t l_{1}$, where $t \in T^{a}$ and $l_{1} \in L_{1}$. If $f_{1} \in F_{1}$, then $\left(t \cdot l_{1}\right) f_{1}=t f_{1} l_{1}=f_{1}\left(t l_{1}\right)$ since $t$ is central, and $f_{1} l_{1}=l_{1} f_{1}$ since $l_{1} \in L_{1}$. Thus $x$ commutes with $F_{1}$, and $x$ commutes with $T^{a}$.

Conversely, if $x$ belongs to the connected component of the centralizer of $T^{a} \cup F_{1}$, then $x \in G_{2}$, so that $x=\operatorname{tg}_{3}$. Moreover, since $x$ commutes with $F_{1}$, we have $x f_{1}=f_{1} x$, so that $t g_{3} f_{1}=f_{1} t g_{3}=t f_{1} g_{3}$, so that $g_{3} f_{1}=f_{1} g_{3}$, thus $g_{3} \in L_{1}$, and $x \in T^{a} \times L_{1}$, that is, $x \in L$.

LEMMA 4.3. Let $G$ be compact, connected Lie group and $L$ a subgroup of $G$, such that $L$ is the centralizer in $G$ of a toral subgroup $T^{a}$. Then there exists an $x \in T^{a}$ such that (1) $L$ is the connected component of the centralizer of $x$ in $G$, and (2) $\operatorname{Ad}(x)$ is of odd order.

Proof of (1). $T^{a} \subset T^{b}$, where $T^{b}$ is a maximal torus of $G$. If $\theta_{i}$, $i=1, \cdots, m$, are the root forms, or angular parameters of $T^{b}$, and $R^{b}$ is the covering space of $T^{b}$, then $R^{a}$, the covering space of $T^{a}$, is characterized by $R^{a}=\left\{x: \theta_{i}(x)=0, i=1, \cdots, m^{\prime}, m^{\prime} \leqq m\right\}$, where $\theta_{i}, i=1, \cdots, m^{\prime}$, are angular parameters of $T^{a}$. If $x \in T^{a}$, and $Z(x)$ denotes the connected component of the centralizer of $x$ in $G$, then $L \subset Z(x)$. Moreover $Z(x)$ is minimal if $\theta_{i}(x) \neq 0, i=m^{\prime}+1, \cdots, m$. But each plane $\theta_{i}(x)=0, i=m^{\prime}+1, \cdots, m$, determines a hyperplane of $R^{a}$, and the union of these finitely many hyperplanes cannot be all of $R^{a}$, thus there exist $x \in R^{a}, \theta_{i}(x) \not \equiv 0, i=m^{\prime}+1, \cdots, m$.

Proof of (2). Given any Euclidean space $R$, the set of elements with coordinates whose denominators are all odd is dense in $R$, because the set of points with rational coordinates is dense in $R$. And 
if $x=\left(r_{1}, \cdots, r_{n}\right)$ where $r_{i}=p_{i} / q_{i}, p_{i}, q_{i}$ relatively prime, is a point with rational coordinates and

$$
x_{m}=\left(\frac{2 m p_{1}}{2 m q_{1}+1}, \frac{2 m p_{2}}{2 m q_{2}+1}, \ldots, \frac{2 m p_{n}}{2 m q_{n}+1}\right),
$$

then $\lim _{m \rightarrow \infty} x_{m}=r$.

Now let $\bar{\theta}_{i}, i=1, \cdots, b$, be a basis for $R^{b}$, consisting of root vectors. Thus if $x \in R^{b}$, then $\left\langle\bar{\theta}_{i}, x\right\rangle=\theta_{i}(x)$ defines an inner product on $R^{b}$, and if $x=\sum x_{i} \bar{\theta}_{i},\left\langle\bar{\theta}_{i}, x\right\rangle=x_{i}$ so that all $\theta_{i}(x) \equiv 0 \bmod 1$, if $x$ has integral coordinates in this basis, that is, $\operatorname{Ad} x=1$.

Now if $x \in T^{a}$, such that $Z(x)=\mathscr{L}$, then it does not lie on any of the planes $\theta_{i}(x)=0, i=m^{\prime}+1, \cdots$, which is a closed set, and there exists a point $z$ with rational coordinates, with odd denominators, arbitrarily close to $x$, so that $Z(x)=\mathcal{L}$. But $\operatorname{Ad}\left(z^{k}\right)=1$, where $k=$ the least common multiple of the denominators of the coordinates of $z$. And, $\operatorname{Ad}\left(z^{k}\right)=\operatorname{Ad}^{k}(z)$.

Combining the last two lemmas, we have that since $L$ is the centralizer of $x \in T^{a}$, such that $\operatorname{Ad}(x)$ is of odd order and $L$ is the connected component of the centralizer of $F_{1}$, then $L$ is the connected component of the centralizer, in $G$, of the group generated by $x$ and $F_{1}$, whose adjoint is of odd order.

\section{BIBLIOGRAPHY}

1. A. Borel and J. deSiebenthal, Les sous-groupes fermés de rang maximum des groupes de Lie clos, Comment. Math. Helv. 23 (1949), 200-221.

2. A. Frölicher, Zur Differentialgeometrie der komplexen Strukturen, Math. Ann. 129 (1955), 50-95.

3. R. Hermann, Compact homogeneous almost complex spaces of positive characteristic, Trans. Amer. Math. Soc. 83 (1956), 471-481.

4. H. C. Wang, Closed manifolds with homogeneous complex structure, Amer. J. Math. 76 (1954), 1-32.

Lake Forest College AND New York UNIVERSITY 